\title{
Previsão de value-at-risk para o mercado de criptomoedas usando modelos EGARCH com regimes markovianos
}

\author{
(Forecasting value-at-risk for the cryptocurrency market using \\ Markov-switching EGARCH models)
}

\section{Paulo Fernando Marschner \\ Paulo Sergio Ceretta}

\begin{abstract}
Resumo Essa pesquisa teve como objetivo compreender o comportamento volátil em seis criptomoedas de grande representatividade. Para tal, foram estimados modelos EGARCH com e sem regimes markovianos, combinados com diferentes distribuições de probabilidade estatística e testada a capacidade preditiva dos melhores modelos resultantes dessa combinação por meio da previsão do value-at-risk. Esse estudo demonstrou que os log-retornos diários das criptomoedas analisadas apresentam claramente mudanças de regime em sua dinâmica de volatilidade. Na análise in-sample, o modelo com mudança de regime confirma a existência de dois estados: o primeiro caracterizado por um maior efeito ARCH e menos afetado por assimetrias, enquanto o segundo revela maior efeito da chegada de informações, ou seja, é mais sensível à choques assimétricos. $\mathrm{Na}$ análise out-sample, as previsões de valor em risco dos modelos com mudança de regime superam claramente os modelos de regime único em um $\alpha=1 \%$.
\end{abstract}

Palavras-chave: Criptomoedas; Volatilidade; Mudança de regime; Value-at-risk.

Código JEL: G10, G17.

\begin{abstract}
This study aims to understand the volatile behavior of six highly representative cryptocurrencies. To do so, EGARCH and Markov-switching EGARCH models were estimated, combined with different distributions of statistical probability. The predictive capacity of the best models resulting from these combinations were tested by predicting the value-at-risk. The daily returns of the cryptocurrencies clearly show regime changes in their volatility dynamics. In the in-sample analysis, the regime change model confirms the existence of two states: the first characterized by a greater ARCH effect and less affected by asymmetries, while the second reveals a greater effect of the arrival of information, that is, it is more sensitive to asymmetric shocks. In the out-of-sample analysis, the value-at-risk predictions of the regime change model clearly exceed the single-regime model by the extreme quantile of $1 \%$.
\end{abstract}

Keywords: Cryptocurrencies; Volatility; Regime change; Value-at-Risk.

JEL Code: G10, G17.

Submitted on March 11, 2020. Revised on July 4, 2020. Accepted on August 6, 2020. Published online in September 2020. Editor in charge: Alan de Genaro.

${ }^{\dagger}$ Universidade Federal de Santa Maria, Brazil: paul ofernandomarschner@hotmail.com

*Universidade Federal de Santa Maria, Brazil: ceretta10@gmail.com 


\section{Introdução}

As perturbações em grandes escalas ocorridas pela crise financeira global fizeram com que alguns investidores perdessem a confiança no sistema monetário convencional. De acordo com Dyhrberg (2016) a crise financeira facilitou o surgimento de uma nova classe de ativos conhecidos como criptomoedas. Em dezembro de 2019, o valor total do mercado de criptomoedas havia excedido 290 bilhões e o número total de criptomoedas já ultrapassava 2.000. O crescimento acentuado desse mercado decorre da visão dos investidores de que a moeda digital pode ser considerada um abrigo contra turbulências econômicas e financeiras devido às suas baixas correlações com outros ativos do mercado financeiro (Bouri et al., 2017).

Apesar desses novos ativos serem considerados uma grande inovação financeira (Bouoiyour et al., 2015), um aspecto essencial a ser explorado é sua volatilidade, ou seja, a intensidade e a frequência das oscilações em seu preço (Silva et al., 2019). Alguns autores indicam que a volatilidade excessiva desse mercado é uma grande preocupação (Yermack, 2015; Vandezande, 2017). De acordo com Bouoiyour e Selmi (2016), a volatilidade do mercado de criptomoedas ainda não foi suficientemente estudada, e devido aos seus altos níveis, a previsão associada a estes ativos se constitui uma tarefa ainda mais desafiadora quando comparada à outras séries precificadas (Yermack, 2015). Devido a essas circunstâncias, o estudo das criptomoedas e o uso das técnicas de previsão são duas agendas importantes da pesquisa em finanças (Peng et al., 2018).

Diversos trabalhos têm utilizado inúmeros modelos de heterocedasticidade condicional para modelar a volatilidade das criptomoedas. Chu et al. (2017) encontraram evidências de clusters de volatilidade nas séries de criptomoedas, e indicaram que o modelo Generalized Autoregressive Conditional Heteroscedasticity (GARCH) e seus variantes são eficientes para modelar sua volatilidade. Utilizando modelos GARCH assimétricos, Bouri et al. (2016), Katsiampa (2017) e Baur et al. (2018) encontraram evidências de um efeito alavancagem e um alto grau de persistência no processo de volatilidade. Essas evidências também foram observadas por Catania e Grassi (2017) e Phillip et al. (2018). Trucíos (2019) comparou diversos modelos GARCH com diferentes distribuições de erros e comparou o desempenho destes na estimativa do value-at-risk (VaR), sendo favorável a nova classe de modelos GAS. Trucíos et al. (2019) compararam os modelos GARCH robusto e GAS na estimativa do VaR e Expected Shortfall (ES), sendo favoráveis aos modelos GARCH robusto.

Outros pesquisadores investigaram as características dos retornos das crip- 
tomoedas que podem enviesar as estimativas ou reduzir o poder de previsão de modelos tradicionais como os do tipo GARCH. Barivieira et al. (2017) e Balcome e Fraser (2017) descobriram que os retornos do Bitcoin exibem alguma forma de mudança de regime, sugerindo que os modelos de troca de regime poderiam capturar de forma mais adequada sua dinâmica de volatilidade. Essa possibilidade foi confirmada posteriormente por Ardia et al. (2019) que encontraram fortes evidências de mudanças de regime nos retornos do Bitcoin, mostrando que os modelos Markov-switching GARCH superam as especificações de regime único ao prever o VaR. Silva et al. (2019) demonstraram a existência de dois regimes de volatilidade, um com maior, e outro com menor volatilidade, ambos com forte característica assimétrica. Já Caporale e Zekokh (2019) descobrem que as criptomoedas exibem alta volatilidade e efeito alavancagem em pelo menos um regime, e que os modelos com mudança de regime produzem melhores previsões de VaR.

Dadas essas descobertas, tornam-se essenciais modelos adequados para determinar se mudanças de regime estão presentes na dinâmica de volatilidade das criptomoedas. Embora já existam na literatura aplicações empíricas de mudança de regime nos modelos GARCH (Sajjad et al., 2008) e Asymmetric Power ARCH (APARCH) (Bensaida et al., 2018), existem evidências de que o desempenho de um modelo de mudança de regime tende a ser consideravelmente melhorado se combinado com o modelo Exponential GARCH (EGARCH) proposto por Nelson (1991) (Henry, 2009; Dendramis et al., 2014).

Dessa forma, essa pesquisa testa se os modelos Markov-switching EGARCH (MS-EGARCH) capturam mudanças de regime na dinâmica de volatilidade das criptomoedas e superam as especificações EGARCH de regime único. A análise empírica utiliza dados de seis criptomoedas com grande representatividade no mercado no período de agosto 2017 a setembro de 2018. Como as criptomoedas apresentam violações extremas dos pressupostos de normalidade dos erros e volatilidade excessiva foram consideradas várias distribuições de erros e especificações para até dois regimes, estendendo trabalhos anteriores (Katsiampa, 2017; Peng et al., 2018; Trucíos, 2019). Além disso, a capacidade preditiva desses modelos foi avaliada por meio da previsão do VaR. Em termos de risco, o mercado de criptomoedas foi pouco explorado, tendo a maioria dos estudos analisado apenas o Bitcoin (Chan et al., 2017; Osterrieder e Lorenz, 2017; Trucíos, 2019), e Trucíos et al. (2019) em um número de maior de criptomoedas.

Sendo assim, essa pesquisa amplia a literatura existente de três formas. Primeiro, por analisar um número maior de criptomoedas apresenta um panorama maior desse mercado, tendo em vista que a maioria dos estudos anali- 
saram prioritariamente o Bitcoin. Segundo, considera modelos com mudança de regime, capturando novas informações sobre a dinâmica de volatidade desses ativos. Finalmente, testa os melhores modelos estimados em termos de risco. Os resultados além de indicarem que os modelos com mudança de regime conseguem acomodar com mais precisão às propriedades dos retornos financeiros e das dinâmicas presentes na volatilidade do mercado de criptomoedas, indicam que esses modelos superam as especificações de regime único na previsão do $\mathrm{VaR}$ em um menor intervalo de confiança. Esses resultados podem ser úteis para minimizar a incerteza e o risco associado aos investimentos no mercado de criptomoedas tornando as decisões de investimento mais realistas.

\section{Modelagem econométrica da volatilidade}

A volatilidade pode ser definida como a variabilidade do preço de um ativo ao longo do tempo. Uma das principais características da volatilidade é que ela não é diretamente observável (Tsay, 2013). Apesar disso, a volatilidade apresenta algumas características estilizadas comuns aos retornos financeiros que podem ser detectadas (Tsay, 2010). As principais características estilizadas relatadas na literatura indicam agrupamentos de volatilidade (clusters) (Mandelbrot, 1963; Liu, 2000), distribuições com caudas mais pesadas do que a distribuição normal, comportamento não linear, dependências não temporais e volatilidade não constante ao longo do tempo (Tsay, 2010; Francq e Zokaian, 2010).

Em trabalho seminal, Engle (1982) propôs o modelo Autoregressive Conditional Heteroscedasticity $(\mathrm{ARCH})$ onde a variância condicional pode ser modelada através de uma função quadrática das observações passadas. $\mathrm{O}$ modelo GARCH desenvolvido por Bollerslev (1986), representa a primeira e mais significativa evolução dos modelos heterocedásticos ao propor que a volatilidade condicional fosse função não apenas dos quadrados dos erros passados, mas também dos seus próprios valores.

Embora o GARCH seja eficiente para modelar a volatilidade, é limitado ao considerar que choques positivos e negativos impactem a volatilidade da mesma forma, desconsiderando que as inovações podem impactar de forma assimétrica a volatilidade (Wennström, 2014). O modelo EGARCH proposto por Nelson (1991) inovou aos modelos de volatilidade na medida em que foi capaz de modelar o comportamento assimétrico que as inovações causam na volatilidade dos ativos financeiros. Autores como Laurent et al. (2012) enfatizam que os modelos que incorporam os efeitos da assimetria na volatilidade das séries financeiras como o EGARCH geram menores erros de previsão fora da amostra em comparação com o GARCH tradicional, e por isso tendem a 
ser mais eficientes.

$\mathrm{Na}$ forma simplificada o modelo EGARCH $(1,1)$ pode especificado da seguinte forma:

$$
r_{t}=\mu_{t}+\varepsilon_{t}
$$

com

$$
\varepsilon_{t} \sim N\left(0, h_{t}\right)
$$

e

$$
\log \left(h_{t}\right)=\omega+\alpha\left[\left|\frac{\varepsilon_{t-1}}{\sqrt{h_{t-1}}}\right|-\sqrt{2 / \pi}\right]+\beta \log \left(h_{t-1}\right)+\delta \frac{\varepsilon_{t-1}}{\sqrt{h_{t-1}}} .
$$

Em (1) a construção logarítmica garante que a variação condicional estimada $h_{t}$ seja positiva, evitando a necessidade de restrições de não-negatividade em alguns parâmetros, normalmente usadas na estimativa de modelos GARCH. Além disso, como $\widehat{\delta}$ é tipicamente negativo em sinal, uma inovação negativa, $\varepsilon_{t}<0$, gera mais volatilidade do que uma inovação positiva de igual magnitude (Francq e Zokaian, 2010). No modelo EGARCH, a inovação pode ser tratada como uma medida coletiva de notícias sobre os preços dos ativos que chegam ao mercado no período $t-1$ a $t$. Considerando que as informações sejam mantidas constantes no tempo $t-2$ e antes, Engle e Ng (1993) descrevem a relação entre $\varepsilon_{t-1}$ e $h_{t}$ como a curva de impacto das notícias:

$$
h_{t}= \begin{cases}A \exp \left[\frac{\delta+\alpha}{\sigma} \varepsilon_{t-1}\right] & \text { para } \varepsilon_{t-1}>0 \\ A \exp \left[\frac{\delta-\alpha}{\sigma} \varepsilon_{t-1}\right] & \text { para } \varepsilon_{t-1}<0\end{cases}
$$

Em (2), $A=\sigma^{2 \beta} \exp [\omega-\alpha \sqrt{2 / \pi}]$. Devido a estrutura da curva de impacto das notícias $\delta<0$ fará com que a inclinação da curva no segmento $\varepsilon_{t-1}>0$ seja mais íngreme do que a inclinação correspondente no segmento $\varepsilon_{t-1}<0$; portanto, a volatilidade responde assimetricamente ao sinal do choque. Essas características tornam o modelo EGARCH um dos principais modelos de volatilidade dado seu baixo esforço computacional, baixo número de parâmetros e sua capacidade de capturar clusters de volatilidade e o comportamento não-constante da variância condicional (Hansen e Lunde, 2005).

Além do EGARCH, outras extensões foram desenvolvidas, incluindo o modelo GJR-GARCH de Glosten et al. (1993), o Threshold GARCH de Zakoian (1994), o Nonlinear GARCH de Higgins e Bera (1992), o APARCH de Ding et al. (1993), entre outros. Embora tais modelos apresentem desempenho na modelagem de séries financeiras, sabe-se que estas tendem a apresentar mudanças de comportamento ao longo do tempo que podem caracterizar diversos regimes. Um exemplo é quando o mercado se encontra 
em regimes de recessão e expansão. Por isso, a principal desvantagem dos modelos GARCH de regime único é que eles tendem a superestimar a persistência de um choque diante de uma mudança não parametrizada de regime (Lamoureaux e Lastrappes, 1990; Cai, 1994; Hamilton e Susmel, 1994).

\subsection{O modelo Markov-switching EGARCH}

O trabalho de Hamilton (1989) foi uns dos mais importantes para o desenvolvimento de modelos com mudança de regime, mostrando que nestes apenas alguns parâmetros são dependentes do estado. A característica principal dos modelos com mudança de regime é a possibilidade de alguns, ou todos os parâmetros mudarem em cada regime (ou estado) de acordo com um processo de Markov. Algumas aplicações em modelos de volatilidade incluem os trabalhos de Henry (2009), Dendramis et al. (2014) e Bensaida et al. (2018), por exemplo.

O trabalho de Henry (2009) foi um dos pioneiros a modelar um EGARCH com mudança de regime. De acordo com o autor, um modelo EGARCH com mudança de regime pode ser especificado da seguinte forma:

$$
r_{t}=\mu_{i, t}+\varepsilon_{t}
$$

com

$$
\varepsilon_{t} \sim N\left(0, h_{i, t}\right)
$$

$\mathrm{e}$

$$
\log \left(h_{i, t}\right)=\omega+\alpha_{i}\left[\left|\frac{\varepsilon_{i, t-1}}{\sqrt{h_{i, t-1}}}\right|-\sqrt{2 / \pi}\right]+\beta_{i} \log \left(h_{i, t-1}\right)+\delta \frac{\varepsilon_{i, t-1}}{\sqrt{h_{i, t-1}}} .
$$

Esse modelo permite a existência de dois estados, $i$, indexados por uma variável latente não observada $S_{t}$ que assume o valor de 0 ou 1, dependendo do estado. Seguindo Hamilton (1989), supõe-se que $S_{t}$ siga um processo de Markov de dois estados com uma matriz de probabilidade de transição fixa $P$ :

$$
P=\left[\begin{array}{cc}
p^{00} & 1-p^{11} \\
1-p^{00} & p^{11}
\end{array}\right]
$$

Em (4), $p^{11}=\operatorname{Pr}\left(S_{t}=i \mid S_{t-1}-i\right)$ para $i=0,1$. Implicitamente, $S_{t}$ depende das realizações passadas de $r$ e $S$ apenas através de $S_{t-1}$. As probabilidades de transição são inicialmente consideradas constantes e especificadas como:

$$
p^{00}=\frac{\exp \left\{\theta_{0}\right\}}{1+\exp \left\{\theta_{0}\right\}} \quad \text { e } \quad p^{11}=\frac{\exp \left\{\gamma_{0}\right\}}{1+\exp \left\{\gamma_{0}\right\}}
$$


Como a variação condicional depende de todo o histórico dos dados em um modelo com mudança de regime (Cai, 1994; Hamilton e Susmel, 1994), Gray (1996) argumenta que é possível construir uma medida de $h_{t}$ que não seja dependente do caminho. De modo que $h_{t}=E\left[r_{t}^{2} \mid \Omega_{t-1}\right]-E\left[r_{t} \mid \Omega_{t-1}\right]^{2}$ que produz:

$$
h_{t}=p^{00}\left(\mu_{0 t}^{2}+h_{0 t}\right)+\left(1-p^{00}\right)\left(\mu_{1 t}^{2}+h_{1 t}\right)-\left[p^{00} \mu_{0 t}+\left(1-p^{00}\right)\left(\mu_{1 t}\right)\right]^{2},
$$

onde a medida da variação condicional depende das informações disponíveis, mas agregada entre os regimes fornece um modelo de volatilidade independente do caminho. Ou seja, cada variação condicional depende apenas do regime, e não de toda o percurso do processo. Usando (6) no lugar de $h_{i, t-1}$ em (3), tem-se:

$$
r_{t}=\mu_{i, t}+\varepsilon_{t}
$$

com

$$
\varepsilon_{t} \sim N\left(0, h_{i, t}\right)
$$

$\mathrm{e}$

$$
\begin{aligned}
\log \left(h_{i, t}\right) & =\omega_{i}+\alpha_{i}\left[\left|\frac{\varepsilon_{t-1}}{\sqrt{h_{t-1}}}\right|-\sqrt{2 / \pi}\right]+\beta_{i} \log \left(h_{t-1}\right)+\delta_{i} \frac{\varepsilon_{i, t-1}}{\sqrt{h_{t-1}}} \\
h_{t} & =p^{00}\left(\mu_{0 t}^{2}+h_{0 t}\right)+\left(1-p^{00}\right)\left(\mu_{1 t}^{2}+h_{1 t}\right)-\left[p^{00} \mu_{0 t}+\left(1-p^{00}\right)\left(\mu_{1 t}\right)\right]^{2}
\end{aligned}
$$

A restrição $\left|\beta_{i}\right|<1$ para todos os regimes $i$, elimina a possibilidade de volatilidade explosiva ou não estacionária. A curva de impacto das notícias também considera os regimes:

$$
h_{t}= \begin{cases}A_{i} \exp \left[\frac{\delta+\alpha_{i}}{\sigma} \varepsilon_{t-1}\right. \\ A_{i} \exp \left[\frac{\delta-\alpha_{i}}{\sigma} \varepsilon_{t-1}\right] & \text { para } \varepsilon_{t-1}>0, \\ \text { para } \varepsilon_{t-1}<0 .\end{cases}
$$

$\operatorname{Em}(8), A_{i}=\sigma^{2 \beta i} \exp \left[\omega_{i}-\alpha_{i} \sqrt{2 / \pi}\right]$ para regimes $i=1,2, \ldots$. Da mesma forma, o grau de resposta assimétrica às notícias dependerá do regime a menos que $\alpha_{0}=\alpha_{1}$, e $\delta_{0}=\delta_{1}$. Considerando essas características o modelo MS-EGARCH possui flexibilidade suficiente para capturar a dependência do regime no impacto, persistência e resposta assimétrica a um choque.

\section{Dados e método}

Os dados utilizados correspondem as séries de fechamento diário das seis criptomoedas com maior negociabilidade e representatividade: Bitcoin, Bit- 
Tabela 1

Dados do mercado de criptomoedas

\begin{tabular}{lrrrrr}
\hline criptomoeda & $\begin{array}{r}\text { valor de mercado } \\
\text { (US\$) }\end{array}$ & preço (US\$) & volume (24h)(US\$) & $\begin{array}{r}\text { fornecimento } \\
\text { circulante }\end{array}$ & mudança (24h) \\
\hline Bitcoin & $112.665 .035 .864,00$ & $6.516,39$ & $4.306 .910 .115,00$ & 17.289 .475 & $1,40 \%$ \\
Ripple & $22.207 .316 .359,00$ & 0,56 & $1.937 .430 .759,00$ & 39.870 .907 .279 & $23,67 \%$ \\
Ethereum & $22.067 .543 .088,00$ & 215,93 & $1.804 .761 .332,00$ & 102.196 .650 & $2,81 \%$ \\
Bitcoin Cash & $7.769 .221 .291,00$ & 447,30 & $351.178 .810,00$ & 17.369 .275 & $2,17 \%$ \\
EOS & $4.926 .896 .462,00$ & 5,44 & $673.279 .297,00$ & 906.245 .118 & $5,29 \%$ \\
Stellar & $4.802 .184 .116,00$ & 0,26 & $103.002 .415,00$ & 18.789 .946 .755 & $7,82 \%$ \\
\hline
\end{tabular}

Nota: As séries temporais foram coletadas 09:00 horas do dia 26 de setembro de 2018 no site: https : //coinmarketcap.com/.

coin Cash, Ethereum, Ripple, EOS e Stellar, coletados na página do CoinMarket. $O$ período coletado vai de 02 de agosto de 2017 a 25 de setembro de 2018, totalizando 420 observações. É possível observar por meio da Tabela 1 informações sobre cada uma das criptomoedas. Em 26 de setembro de 2018 haviam duas mil e três criptomoedas negociadas nesse mercado as quais representavam um valor de mercado de aproximadamente US\$ 215.526.901.785,00. Essas seis criptomoedas representavam 73,43\% da capitalização total de mercado e 50,32\% do volume negociado. Este fato garante uma grande representatividade da amostra considerada para análise.

É possível observar grandes diferenças entre cada uma das criptomoedas. Ripple era a criptomoeda com maior número de unidades em circulação e maior oscilação de preço (aproximadamente 24\%). Bitcoin possui o maior preço e volume, porém o menor número de unidades em circulação, possivelmente por ser a moeda mais antiga, o que dificulta a mineração de novas unidades. Apesar disso, o Bitcoin possuía um domínio de 52,3\% de todo esse mercado.

Inicialmente, para corrigir possíveis problemas de não-estacionariedade as séries originais foram transformadas em log-retornos. A amostra é dividida em duas partes, o primeiro subconjunto refere-se ao período denominado insample (dados de 02 agosto de 2017 a 17 de junho de 2018) que foi tomado como amostra de estimação. O segundo subconjunto se refere ao período fora da amostra denominado out-sample (dados de 18 de junho a 25 de setembro de 2018) em que as previsões de VaR através dos modelos concorrentes são realizadas e avaliadas.

Como destacado pela literatura, as criptomoedas exibem oscilações que violam a suposição de normalidade e por isso, pode-se supor que os erros seguem uma distribuição não gaussiana (Peng et al. 2018). Portanto, os modelos EGARCH e MS-EGARCH serão estimados assumindo quatro distribuições: i) normal, ii) normal assimétrica, iii) Student- $t$, e iv) Student- $t$ as- 
simétrica. Embora exista uma variedade de distribuições a serem assumidas a um modelo de volatilidade, as distribuições do tipo Student's são suficientes para um bom ajuste à cauda pesada dos dados financeiros (Sun e Zhou, 2014). Essa combinação resultou em um total de 8 modelos concorrentes que foram selecionados a partir do Critério de Informação Akaike (AIC) (Akaike, 1973) que utiliza o valor maximizado da função de verossimilhança para o modelo estimado, o número de parâmetros e o tamanho da amostra. Nesse critério, quanto menor o valor, melhor, ou seja, serão selecionados como melhores aqueles modelos que apresentarem menor valor de AIC. O AIC é particularmente recomendado para penalizar séries com mais de 100 observações, pois nesse caso, critérios como o de Schwarz (BIC) podem ser falhos (Emiliano et al., 2014). Após a verificação dos modelos que melhor se ajustam em cada série foram utilizados os melhores de cada grupo para estimação da volatilidade condicional.

Para avaliar a utilidade dos métodos de previsão de volatilidade foram analisados seus desempenhos por meio de critérios econômicos em termos de análise de risco. O VaR foi adotado por profissionais, investidores e reguladores como um mecanismo para medir o risco de mercado dos ativos financeiros. Uma especificação válida do VaR para qualquer função de distribuição de probabilidade pode ser demonstrada através de termos probabilísticos. Se $R_{t}$ representa uma série de retornos de um ativo financeiro no tempo $t$, o $\operatorname{VaR}(\alpha)$ no percentil $(1-\alpha)$ é definido por $\operatorname{Pr}\left(R_{t} \leq \operatorname{VaR}_{t}(\alpha)\right)=\alpha$, que mede a probabilidade de $R_{t}$ ser menor, ou igual a $\operatorname{VaR}_{t}(\alpha)$ dado um nível de significância $\alpha \in(0,1)$. Em termos probabilísticos, o VaR é apenas um quantil da distribuição de perda (Mcneil et al., 2005). Como o VaR mede por meio de um número positivo a pior perda em um dado nível de significância $\alpha \in(0,1)$, a taxa de retorno $R_{t}^{*}$ resultante neste menor valor da carteira, $W_{t}^{*}$ pode ser dado por $W_{t}^{*}=W_{0}\left(1+R_{t}^{*}\right)$.

A estimação do VaR pode ser simplificada e produzir medidas mais precisas quando se utiliza uma abordagem paramétrica para sua estimação (Jorion, 2007). Neste caso, o VaR pode ser considerado uma função determinística da volatilidade de um ativo financeiro, representando uma aplicação natural dos modelos de volatilidade (Giot e Laurent, 2004). Para estimação do VaR através desta abordagem é necessário que a função de distribuição geral $f(w)$, seja transformada em uma função de distribuição padrão $\varnothing\left(\varepsilon_{t}\right)$, onde $\varepsilon_{t}$ tenha média zero, e desvio padrão unitário. Para tal, $w^{*}$ associa-se com o retorno de corte $R_{t}^{*}$, que por sua vez, associa-se com o desvio padrão normal $\delta$, da seguinte forma:

$$
1-\alpha=\int_{-\infty}^{w^{*}} f(w) \mathrm{d} w=\int_{-\infty}^{R_{t}^{*}} f\left(r_{t}\right) \mathrm{d} r=\int_{-\infty}^{-\delta} \phi\left(\varepsilon_{t}\right) \mathrm{d} \varepsilon
$$


Em (9), encontrar o VaR é equivalente a encontrar o desvio padrão normal $(\delta)$, de tal modo que a área à esquerda da distribuição seja $1-\alpha$. Ao considerar que $R_{t}=\mu+\varepsilon_{t}$, onde $\varepsilon_{t}$ utiliza uma função de distribuição padrão $\phi$ com média zero e variância $\sigma_{t}^{2}$, o VaR pode ser estimado da seguinte forma:

$$
\operatorname{VaR}_{t}(\alpha)=\mu+\sigma_{t} \phi^{-1}(\alpha),
$$

onde $\phi^{-1}(\alpha)$ é o valor do $q$-quantil $(q=1-\alpha)$, da função de distribuição padrão $(\phi), \mu$ representa a média, e $\sigma_{t}$ representa o desvio padrão (volatilidade) do ativo financeiro, que pode ser estimado através de um modelo heterocedástico (Gençay et al., 2003). Finalmente, estimam-se todos os parâmetros para todos os modelos e realizam-se as previsões do VaR para cem passos à frente.

A avaliação das estimativas do VaR (Backtesting) foi amparada em diversos testes propostos pela literatura. $\mathrm{O}$ teste de cobertura incondicional $\left(\mathrm{LR}_{\mathrm{uc}}\right)$, proposto por Kupiec (1995) examina se a taxa de cobertura incondicional é estatisticamente consistente com o nível de confiança prescrito para o modelo de VaR. Sendo $\alpha$ o intervalo de confiança estabelecido no cálculo do VaR, testa-se a hipótese nula de que $H_{0}: f=\alpha$, contra a hipótese alternativa $H_{0}=f \neq \alpha$ onde $f$ é a taxa de falha. Kupiec (1995) propôs o teste baseado na razão de verossimilhança que pode ser empregado a estimativa de uma amostra pontual estatisticamente consistente com o modelo VaR. A estatística do teste é dada por:

$$
\mathrm{LR}=-2 \ln \left[(1-\alpha)^{N=x}\right]+2 \ln \left[(1-f)^{N-x} f^{x}\right] \sim \chi^{2}
$$

O teste segue uma distribuição de qui-quadrado com 1 grau de liberdade. Embora o teste LR uc possa indicar um modelo que sobrestima ou subestima o VaR, o teste é limitado ao determinar se as exceções de VaR são distribuídas aleatoriamente, ou seja, se não correlacionadas ao longo do tempo ( $\mathrm{Su}$ e Hung, 2011). Para superar tal limitação Christoffersen (1998) propôs o teste de cobertura condicional (LRcc) especificado da seguinte forma:

$$
\begin{aligned}
\mathrm{LR}_{\mathrm{cc}} & =\mathrm{LR}_{\mathrm{uc}}+\mathrm{LR}_{\text {ind }} \\
& =-2 \ln \left[\frac{\alpha_{\mathrm{VaR}}^{f}\left(1-\alpha_{\mathrm{VaR}}\right)^{T-f}}{\widehat{\pi}_{01}^{f 01}\left(1-\widehat{\pi}_{01}\right)^{f 00} \widehat{\pi}_{11}^{f 11}\left(1-\widehat{\pi}_{11}\right)^{f 10}}\right] \sim \chi_{(2)}^{2}
\end{aligned}
$$

Em (12), $f_{\mathrm{ij}}$ é o número de observações com valor $i$ seguido de valor $j(i, j=0,1), \widehat{\pi}_{\mathrm{ij}}=P_{r}\left\{\delta_{t}=j \mid \delta_{t-1}=i\right\}, \widehat{\pi}_{01}=f_{01} /\left(f_{00}+f_{01}\right), \widehat{\pi}_{11}=f_{11} /\left(f_{10}+\right.$ $f_{11)}$. O teste tem como hipótese nula que a proporção de violações do VaR 
ocorridas é igual ao número de proporções esperadas (testando $p=\widehat{\pi}$ ) e de que estas violações ocorrem de maneira independente (testando $\widehat{\pi}_{01}=\widehat{\pi}_{11}$ ).

O Teste de Quartil Dinâmico (DQ) proposto por Engle e Manganelli (2004) mede a independência das violações de retorno do VaR. Dada uma variável aleatória $\mathrm{HiT}_{t}=H_{t}-\tau^{*}$, o teste dinâmico de quantis condicionais (DQ) é especificado da seguinte forma:

$$
\mathrm{DQ}=\frac{\left(\mathrm{Hit}_{t}^{\prime} X_{t}\left[X_{t}^{\prime} X_{t}\right]^{-1} X_{t}^{\prime} \mathrm{Hit}_{t}\right)}{\left(T_{\tau}(1-\tau)\right)}
$$

Em (13), o vetor de instrumentos $X_{t}$ pode incluir atrasos de $\mathrm{Hit}_{t}, V_{t}$ e seus atrasos. Desta forma, testa-se a hipótese nula de que $\mathrm{Hit}_{t}$ e $X_{t}$ são ortogonais. Sob sua hipótese nula, a estatística de teste proposto segue uma $X_{q}^{2}$ no qual $q=\operatorname{rank}\left(X_{t}\right)$.

Por fim o desvio absoluto da violação de retornos (AD) proposta por McAleer e Da Veiga (2008) avalia o desempenho da previsão com base na média e no máximo do desvio absoluto, de forma com que valores menores são preferidos acima da amostra da previsão. Se os modelos subestimam o risco, ou seja, possuem muitas falhas, é provável que apresentem valores menores para AD mean e AD maximum. Por outro lado, modelos que superestimam os níveis de risco para violações tendem a gerar valores elevados de ADmean e ADmaximum. A medida proposta por McAleer e Da Veiga (2008) pode ser especificada da seguinte forma:

$$
\mathrm{AD}_{t}=\left|y_{t}-\left(-\mathrm{VaR}_{t}\right)\right| I\left(y_{t}<-\mathrm{VaR}_{t}\right)
$$

Como tal, os modelos com valores pequenos de AD são preferidos somente se estiverem gerando violações independentes na taxa correta $\alpha$.

$\mathrm{Na}$ última etapa é avaliado o desempenho preditivo através de alguma função de perda estatística. Neste trabalho, foram utilizadas três funções de perda para avaliar o desempenho de previsão dos modelos: erro médio absoluto (MAE), média absoluta percentual dos erros (MAPE), e a raiz quadrada do erro-médio (RMSE):

$$
\begin{aligned}
\text { MAE } & =\left(\frac{1}{N}\right) \sum_{i=1}^{N}\left|\left(\gamma_{i}-\widehat{\gamma}_{i}\right)\right| \\
\text { MAPE } & =\left(\frac{1}{N}\right) \sum_{i=1}^{N}\left|\left(\gamma_{i}-\widehat{\gamma}_{i}\right) / \gamma_{i}\right| \times 100 \\
\text { RMSE } & =\sqrt{\left(\frac{1}{N}\right) \sum_{i=1}^{N}\left(\gamma_{i}-\widehat{\gamma}_{i}\right)^{2}}
\end{aligned}
$$


Em (15), (16) e (17), $\gamma_{i}$ é o valor real no período $i$, e $\widehat{\gamma}_{i}$ é a previsão para o período $i$, tal que $i=1,2, \ldots, N$. Um bom modelo é aquele que produz o valor mais próximo do real. Dessa forma, quanto menor forem os valores do MAE, MAPE e RMSE, melhor é a previsão.

\section{Resultados e discussões}

A Figura 1 apresenta a evolução dos valores do preço de fechamento e do log-retorno para cada uma das séries temporais. Por meio da inspeção visual é possível observar que a evolução dos valores de fechamento exibiu um comportamento com grandes oscilações. A partir do fim de 2017 nota-se uma intensa elevação dos preços das criptomoedas o que culminou em valores extremamente elevados no início de 2018 e mantendo certa persistência por aproximadamente um bimestre. Esse comportamento pode estar associado ao início das negociações do Bitcoin na Chicago Board Options Exchange (CBOE) nos Estados Unidos em 10 de dezembro de 2017, e posteriormente na Chicago Mercantile Exchange (CME), fato que pode ter elevado as expectativas de otimismo e confiança dos investidores.

Após esse período percebe-se a existência de variações expressivamente negativas nos preços de fechamento de todos os índices ocasionando grande desvalorização em um período relativamente curto de tempo, especialmente do Ripple, Stellar e Bitcoin Cash. Próximo a setembro de 2018 todas as séries apresentaram a maior desvalorização de preços desde o início do ano. Os logretornos exibem características típicas de séries financeiras como clusters de volatilidade e os padrões de reversão à média.

Na Tabela 2 são apresentadas as estatísticas descritivas, os testes de estacionariedade e de raiz unitária e o teste ARCH-LM. É possível verificar que a média dos log-retornos é próxima a zero. O Bitcoin apresenta maior desvio padrão, seguindo pela Stellar, Bitcoin Cash, EOS, Ripple e Ethereum. Em finanças o desvio padrão é um indicador de risco e quanto mais elevado seu valor, mais arriscado é o ativo. A grande amplitude entre os valores mínimo e máximo fornece uma ideia razoável da grande variabilidade das criptomoedas. O excesso de curtose nas séries do Bitcoin, Ripple, Bitcoin Cash, e Stellar indicam uma característica leptocúrtica nas séries de retornos. Além disso, todas as séries apresentaram assimetria, seja à esquerda ou à direita da média. Como se pode observar, a hipótese nula de normalidade dos logretornos é rejeitada, corroborando fortemente com a premissa de que as criptomoedas exibem oscilações que violam a suposição de normalidade e que os erros seguem uma distribuição não gaussiana (Peng et al. 2018)

Para confirmar que os log-retornos possuem comportamento estacionário foram aplicados os teste de estacionariedade e de raiz unitária Augmented 


\section{Figura 1}
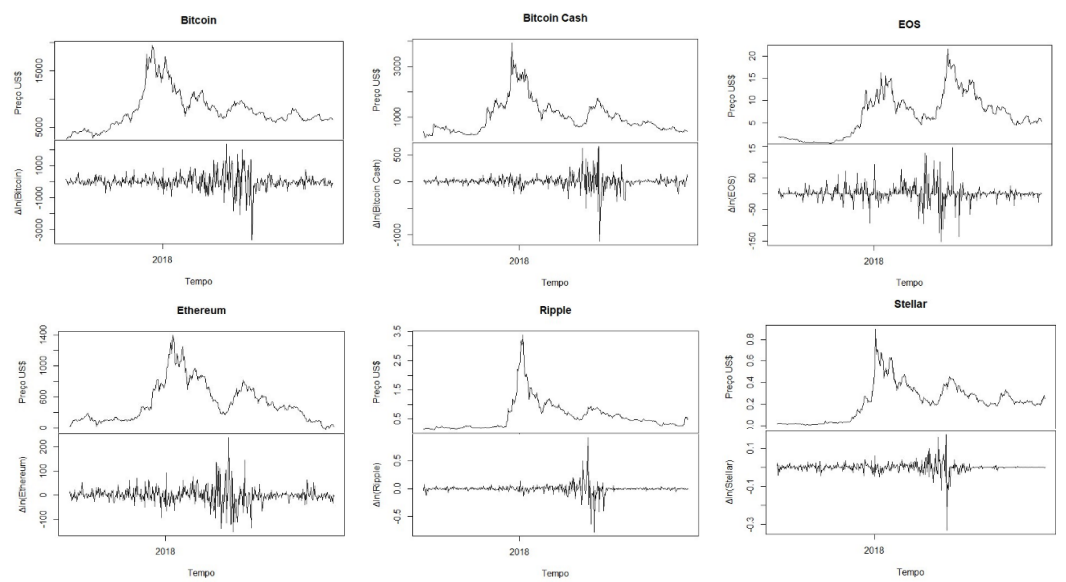

Evolução dos valores do preço de fechamento em nível e log-retorno de cada índice financeiro analisado (período de 2 de agosto de 2017 a 25 de setembro de 2018, dados diários).

Dickey-Fuller (ADF) e Kwiatkowski-Phillips-Schmidt-Shin (KPSS), e constatado que as séries temporais são estacionárias. A heterocedasticidade é um fator salutar na modelagem da volatilidade. Para confirmar tal efeito efetuouse o teste de Multiplicador de Lagrange - ARCH LM proposto por Engle (1982). Os p-valores menores que 0,01, nos dois níveis de defasagens (2 e 6) rejeitam a hipótese nula de homocedasticidade, confirmando a presença de heterocedasticidade.

Após a confirmação da presença de heterocedasticidade foi estimada a volatilidade condicional de cada série para cada um dos oito modelos concorrentes resultantes da combinação dos modelos EGARCH e MS-EGARCH com as quatro distribuições de probabilidade estatística. O AIC é o critério penalizador utilizado para escolher o melhor modelo.

É possível observar uma predominância do modelo EGARCH com a distribuição Student-t assimétrica, com exceção da Ethereum. Já o modelo MSEGARCH apresentou resultados mistos, com predominância de distribuições que consideram um ajuste assimétrico. Isso indica que as distribuições do tipo Student's tendem a acomodar adequadamente as caudas pesadas dos dados financeiros como destacado por Sun e Zhou (2014) e Phillip et al. (2018). Este fato implica que a capacidade de estimar adequadamente a volatilidade das criptomoedas está extremamente vinculada a escolha da distribuição de erros adequada. Seguem descritas nas tabelas 4 e 5 as estimativas para os modelos EGARCH $(1,1)$ e MS-EGARCH $(1,1)$. Ressalta-se que foram apresentados 
Tabela 2

\begin{tabular}{|c|c|c|c|c|c|c|}
\hline \multicolumn{7}{|c|}{ estatística descritiva } \\
\hline & Bitcoin & Bitcoin Cash & EOS & Ethereum & Stellar & Ripple \\
\hline média & $-0,002$ & $-0,005$ & $-0,002$ & $-0,050$ & $-0,006$ & $-0,002$ \\
\hline mediana & $-0,003$ & 0,005 & 0,003 & 0,000 & 0,004 & 0,003 \\
\hline máximo & 2,405 & 0,446 & 0,385 & 0,258 & 0,328 & 0,353 \\
\hline mínimo & $-2,347$ & $-0,431$ & $-0,347$ & $-0,234$ & $-0,666$ & $-0,606$ \\
\hline desvio padrão & 0,171 & 0,093 & 0,090 & 0,057 & 0,095 & 0,083 \\
\hline assimetria & 0,497 & $-0,647$ & $-0,453$ & 0,294 & $-1,193$ & $-1,744$ \\
\hline curtose & 173,22 & 5,195 & 2,968 & 2,454 & 7,113 & 11,587 \\
\hline Jarque-Bera & $0,000^{*}$ & $0,000 *$ & $0,000^{*}$ & $0,000^{*}$ & $0,000 *$ & $0,000^{*}$ \\
\hline \multicolumn{7}{|c|}{ estacionariedade e raiz unitária } \\
\hline $\mathrm{ADF}$ & $-2,561$ & $-2,854$ & $-1,431$ & $-3,033$ & $-2,671$ & $-1,483$ \\
\hline p-valor & 0,010 & 0,004 & 0,001 & 0,002 & 0,007 & 0,002 \\
\hline KPSS & 0,440 & 0,198 & 0,245 & 0,512 & 0,296 & 0,164 \\
\hline p-valor & $>0,100$ & $>0,100$ & $>0,100$ & $>0,100$ & $>0,100$ & $>0,100$ \\
\hline \multicolumn{7}{|c|}{ estatística ARCH-LM } \\
\hline $\operatorname{Lag}(2)$ & 47,595 & 125,525 & 25,452 & 28,019 & 38,849 & 8,201 \\
\hline $\mathrm{p}$-valor & 0,000 & 0,000 & 0,000 & 0,000 & 0,000 & 0,016 \\
\hline $\operatorname{Lag}(6)$ & 62,657 & 129,304 & 67,472 & 81,011 & 43,413 & 81,200 \\
\hline p-valor & 0,000 & 0,000 & 0,000 & 0,000 & 0,000 & 0,000 \\
\hline
\end{tabular}

Estatísticas descritivas dos log-retornos, testes de estacionariedade e raiz unitária, e teste ARCH-LM (período compreendido entre 2 de agosto de 2017 e 25 de setembro de 2018, dados diários). Nota: *Todas as séries de retorno apresentam um comportamento não normal, percebido a partir do teste de normalidade de Jarque-Bera, rejeitados ao nível de $1 \%$ da hipótese de normalidade, considerado pelos p-valores iguais a zero. O Lag (6) foi definido pelo critério de tamanho da série temporal para teste ARCH.

apenas os melhores modelos, de acordo com a Tabela 3.

De forma geral, observa-se que os modelos testados obtiveram um bom ajuste à volatilidade dos retornos pois apresentam parâmetros significativos em todos os casos, ao nível de $1 \%$ de significância. Analisando os modelos EGARCH $(1,1)$ pode-se notar um efeito ARCH $\alpha_{1}$ positivo nas séries do Bitcoin, Bitcoin Cash, EOS, Stellar e Ethereum, e negativo de baixa magnitude no Ripple. Já o efeito assimetria $\alpha_{2}$ embora estatisticamente significativo apresenta baixa ou quase inexistência deste efeito nos modelos de regime único, ou seja, em um único regime choques positivos e negativos tendem a impactar de forma semelhante o mercado de criptomoedas. Pode-se ainda verificar um algo grau de persistência da volatilidade $\beta$, indicando que a volatilidade passada tende a conter informações preditivas sobre volatilidade futura nos modelos de regime único.

Os resultados do modelo de regime único corroboram com os encontrados por Bouri et al. (2016), Katsiampa (2017), Catania e Grassi (2017), Baur et al. (2018), em termos de alavancagem e persistência. Cumpre ressaltar que os resultados destes trabalhos analisaram a volatilidade do Bitcoin a partir 
Tabela 3

Comparação do ajuste de modelos com distribuições alternativas.

\begin{tabular}{|c|c|c|c|c|c|}
\hline \multirow{2}{*}{ série temporal } & \multirow{2}{*}{ modelo de volatilidade } & \multicolumn{4}{|c|}{ distribuição } \\
\hline & & Norm & Snorm & Std & sstd \\
\hline \multirow{2}{*}{ Bitcoin } & EGARCH $(1,1)$ & $-960,91$ & $-959,86$ & $-982,30$ & $-982,37$ \\
\hline & MS-EGARCH $(1,1)$ & $-985,82$ & $-978,55$ & $-980,42$ & $-980,12$ \\
\hline \multirow{2}{*}{ Bitcoin Cash } & $\operatorname{EGARCH}(1,1)$ & $-581,21$ & $-585,09$ & $-632,58$ & $-635,65$ \\
\hline & MS-EGARCH $(1,1)$ & $-636,78$ & $-638,50$ & $-634,88$ & $-640,64$ \\
\hline \multirow{2}{*}{ EOS } & EGARCH $(1,1)$ & $-576,54$ & $-587,43$ & $-630,74$ & $-632,52$ \\
\hline & MS-EGARCH $(1,1)$ & $-637,07$ & $-634,35$ & $-628,88$ & $-633,92$ \\
\hline \multirow{2}{*}{ Ethereum } & EGARCH $(1,1)$ & $-896,70$ & $-896,95$ & $-928,32$ & $-926,90$ \\
\hline & MS-EGARCH $(1,1)$ & $-928,93$ & $-932,68$ & $-926,42$ & $-932,19$ \\
\hline \multirow{2}{*}{ Ripple } & EGARCH $(1,1)$ & $-734,25$ & $-737,67$ & $-804,28$ & $-807,28$ \\
\hline & MS-EGARCH $(1,1)$ & $-804,97$ & $-806,65$ & $-804,27$ & $-801,25$ \\
\hline \multirow{2}{*}{ Stellar } & EGARCH $(1,1)$ & $-563,77$ & $-567,34$ & $-606,01$ & $-606,94$ \\
\hline & MS-EGARCH $(1,1)$ & $-599,50$ & $-599,21$ & $-597,42$ & $-612,81$ \\
\hline
\end{tabular}

Nota: Norm refere-se à distribuição Normal; Snorm refere-se à distribuição Normal Assimétrica; std refere-se à distribuição Student- $t$; e à sstd refere-se à distribuição Student- $t$ assimétrica. Coeficientes em negrito representam os melhores ajustes. Os modelos EGARCH e MSEGARCH foram estimados considerando o número de atrasos, $p$ e $q=1$, o que resulta em estruturas parcimoniosas com alta precisão e poucos parâmetros.

de inúmeros modelos da família GARCH. Já os resultados desse trabalho são amparados em modelos EGARCH, cujas características são favoráveis ao capturar o efeito alavancagem com robustez. Vale ressaltar que a maioria dos modelos aqui estimados assumem distribuições do tipo Student- $t$, semelhante ao trabalho de Phillip et al. (2018) que oferece argumentos favoráveis ao uso desse tipo de distribuição em séries de criptomoedas.

Já os modelos MS-EGARCH $(1,1)$ apresentam um efeito ARCH $\alpha_{1}$ positivo em todas as séries do primeiro regime com exceção do Ripple. No segundo regime, este efeito é negativo além do Ripple também no Bitcoin e EOS. O efeito assimetria $\alpha_{2}$, apresenta um coeficiente negativo no Bitcoin, Ethereum, Ripple e EOS, e positivo no Bitcoin Cash e Stellar no primeiro regime. No segundo regime este efeito mostra-se negativo no Bitcoin e Bitcoin Cash, e positivo nas demais criptomoedas. Isso significa que as criptomoedas são impactadas de formas distintas por choques positivos e negativos dependendo do regime em que estão. Quanto à persistência da volatilidade $\beta$, é possível observar que o Bitcoin apresenta maior persistência no segundo regime, já o Bitcoin Cash e EOS são menos persistentes neste regime, ou seja, há uma memória mais curta em seu processo de volatilidade. Ethereum, Ripple e Stellar não apresentam diferenças significativas entres os regimes. Ao avaliar os modelos com alternância de regime é possível observar que as séries apresentam regimes heterogêneos entre si, ou seja, não seguem um 


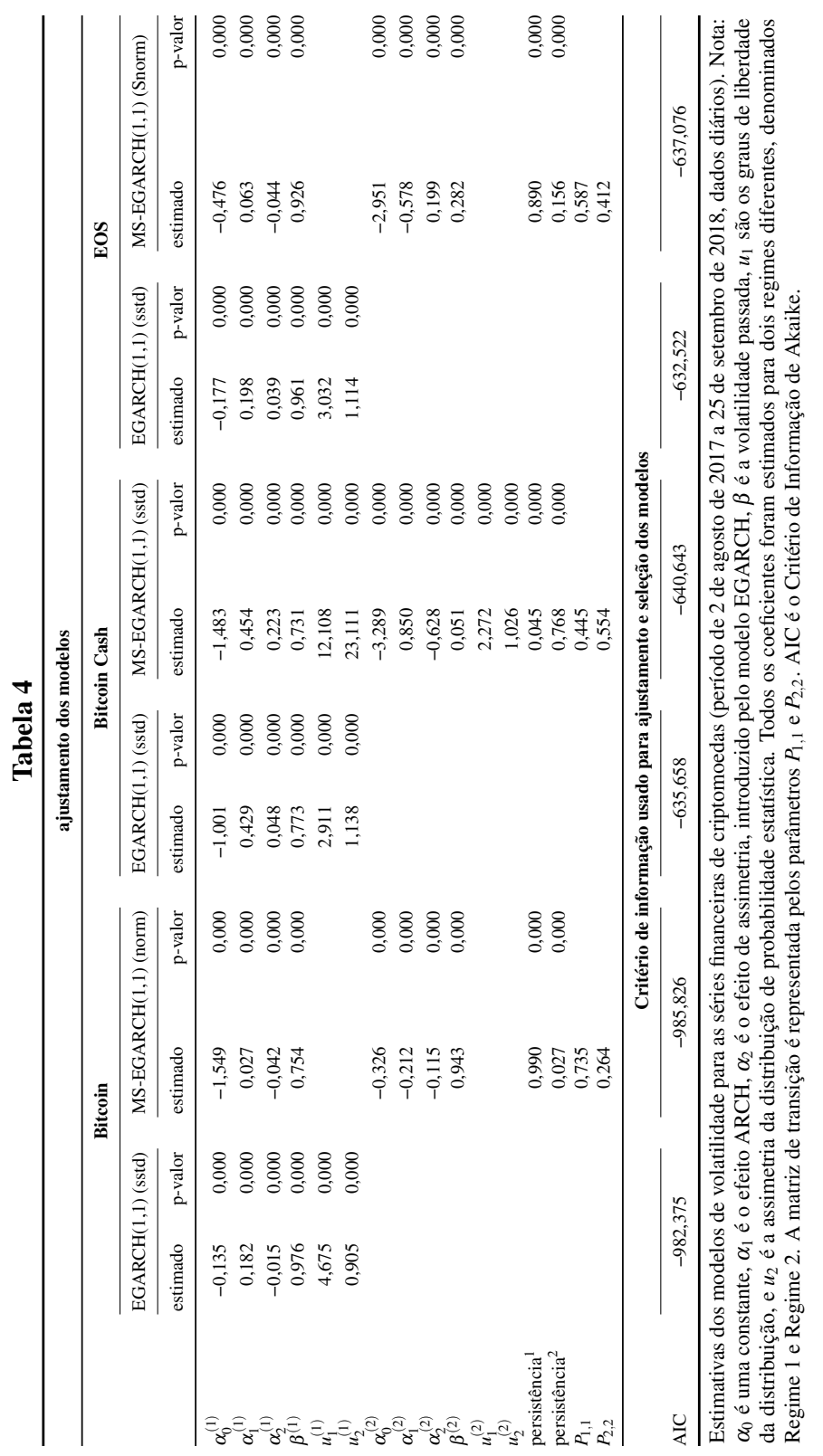




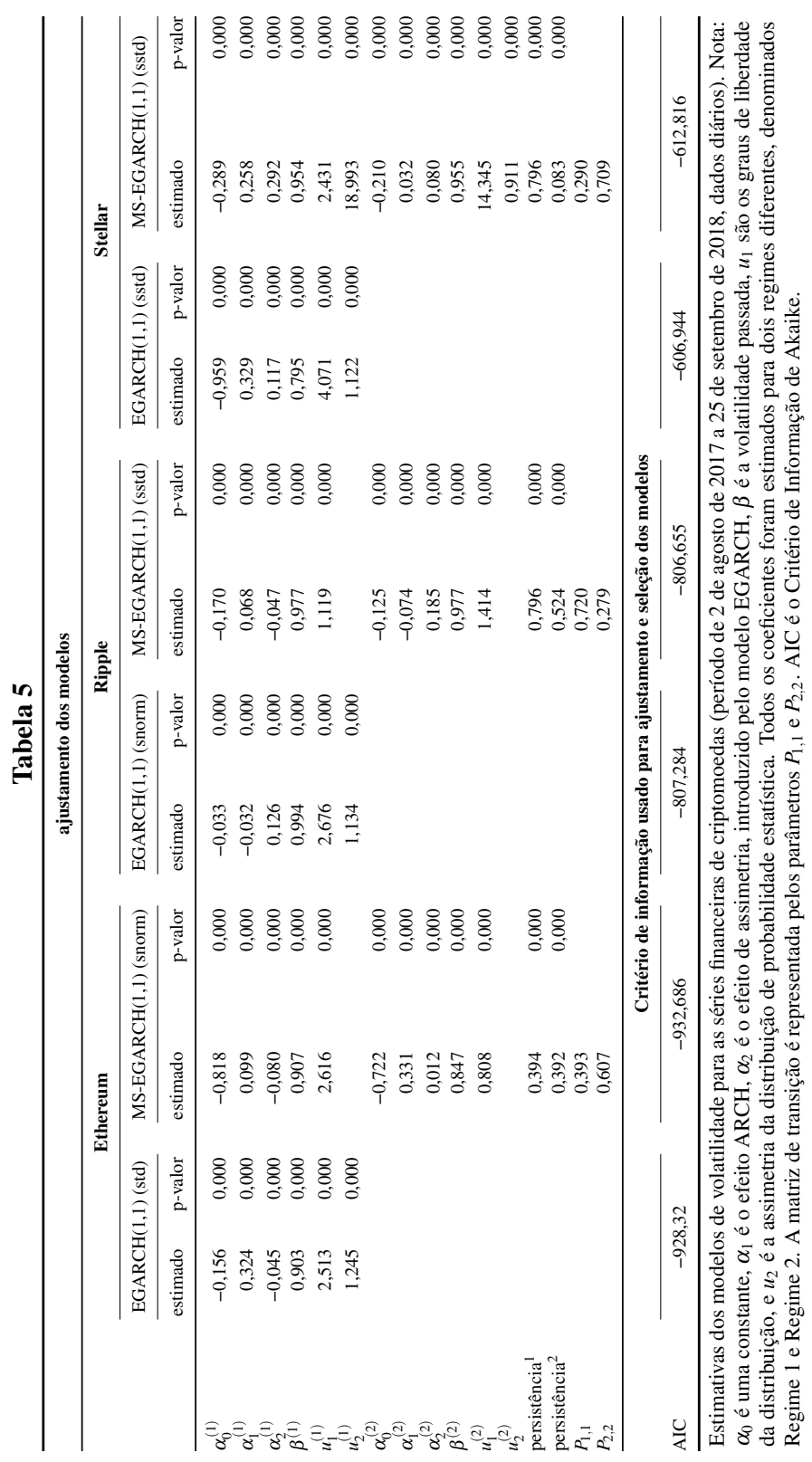


padrão.

Por fim, é possível observar as probabilidades de permanência $P_{1,1}$ ou mudança $P_{2,2}$ de regime. Bitcoin e Ripple apresentam alta persistência em permanecer em um regime. Ethereum e Stellar alta probabilidade de mudar de regime. Bitcoin Cash e EOS apresentam probabilidade de permanência e mudança, mas em ambos os casos as probabilidades não apresentam magnitudes elevadas como nos demais casos. As estimativas confirmaram a existência de dois estados, o primeiro é caracterizado por um maior efeito $\mathrm{ARCH}$, menos afetado por assimetrias e com maior persistência, enquanto o segundo revela maior efeito da chegada de informações, ou seja, é mais sensível a choques assimétricos.

A presença de mudanças de regime nos processos de volatilidade dos logretornos das criptomoedas foi comprovada. Esses resultados vão ao encontro dos obtidos por Silva et al. (2019) que também demostraram a existência de dois regimes, um com maior e outro menor volatilidade, ambos com forte característica assimétrica. Outros estudos que documentaram mudanças de regime incluem os de Barivieira et al. (2017), Balcome e Fraser (2017), Ardia et al. (2019), embora nesses casos, apenas no Bitcoin.

Após esta etapa foram realizadas as previsões de VaR para 100 passos à frente, considerando um $\alpha=1 \%$ e $\alpha=5 \%$. Para testar a qualidade de estimação de VaR segue descrito nas tabelas 6 e 7 o backtesting das estimativas e o desempenho das previsões através das funções de perda.

Em uma estimativa de VaR apropriada, espera-se que a hipótese nula não tenha sido rejeitada em todos os casos, indicando que a proporção estimada de acertos é igual à nominal e que os acertos ocorrem independentemente. Espera-se também que o procedimento produza a menor função de perda quantílica média, o que significa que entre os procedimentos utilizados na comparação, o melhor procedimento relata a menor distância entre os retornos observados e a estimativa de VaR. Os resultados das tabelas 6 e 7 indicam que os testes de Kupiec e Christoffersen não rejeitaram ao nível de significância de 5\% a hipótese nula de predições de VaR corretamente. Ao avaliar o critério de DQ nenhum modelo superou o outro, o que indica que nenhum modelo se mostrou estatisticamente superior ao outro. Os critérios Admean e Admax revelam uma possibilidade maior de ocorrência de falhas no modelos ajustados em maior intervalo de confiança $(\alpha=5 \%)$. De modo geral, as estimativas previram adequadamente a perda esperada em quantis de $5 \%$ e $1 \%$. Assim, os modelos de melhor ajuste podem ser usados para fornecer estimativas aceitáveis de valor em risco. No entanto, esse tipo de procedimento não garante que todas as violações de VaR sejam estatisticamente iguais às expectativas. 


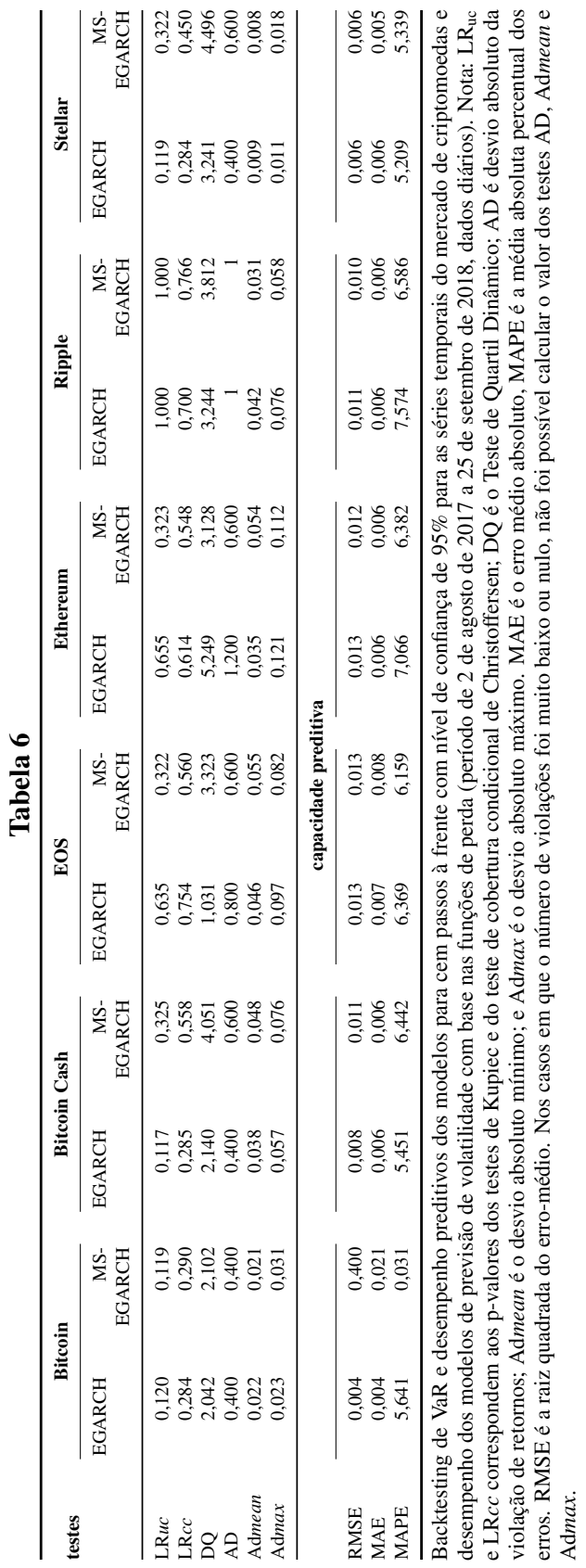




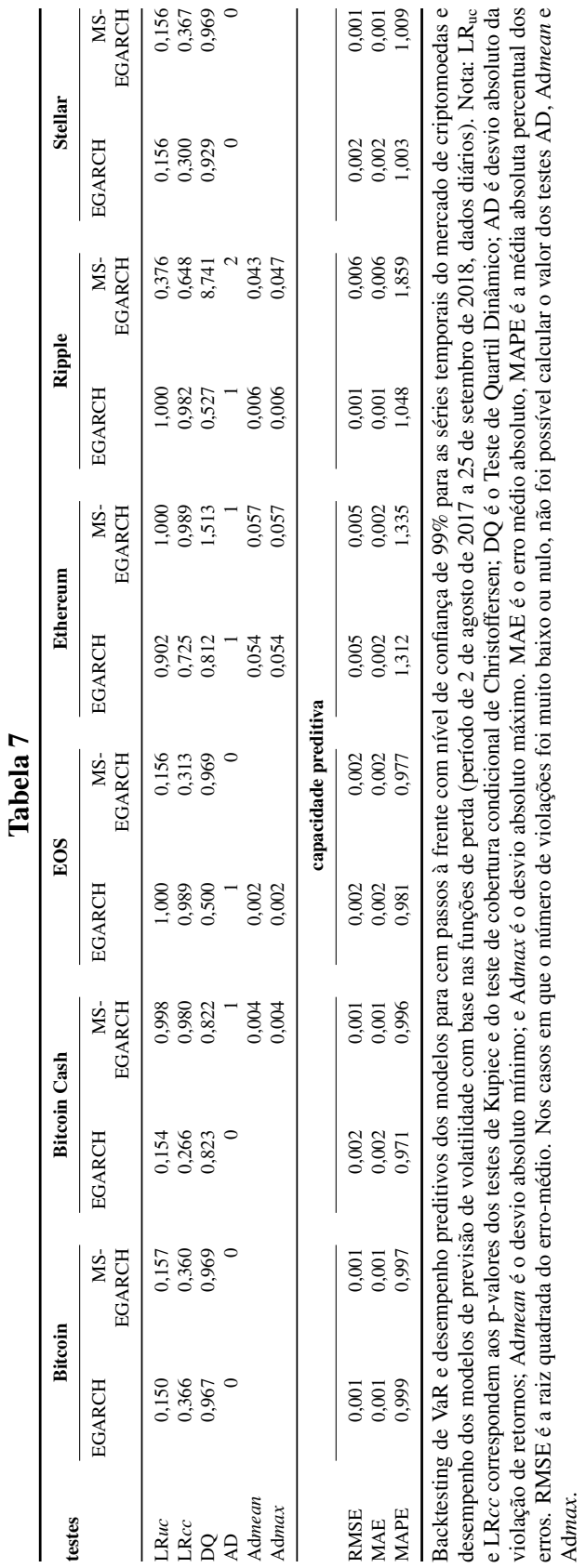


Nas tabelas 6 e 7 também é possível observar a capacidade preditiva dos modelos concorrentes por meio do cálculo das funções de perda. O Bitcoin apresentou melhor capacidade preditiva com o modelo MS-EGARCH, seja ajustado em um intervalo de confiança em $95 \%$ ou 99\%. O Bitcoin Cash apresentou melhor desempenho do modelo EGARCH em 95\% e MS-EGARCH em $99 \%$. O EOS e Stellar não apresentaram mudanças relevantes em $95 \%$, todavia em 99\% o MS-EGARCH apresentou superioridade. Ethereum e Ripple apresentaram melhor desempenho do MS-EGARCH em 95\% e do EGARCH em $99 \%$. Apesar de não existir um modelo de referência, o modelo com alternância de regime parece ser ligeiramente melhor considerando a maioria das funções de perda.

A Figura 2 ilustra a relação entre as perdas observadas e previstas para os 100 passos à frente. É possível observar que em ambos os modelos houve pelo menos uma violação entre o valor real e o previsto em 5\%. A violação em $1 \%$ ocorreu apenas na Ethereum em ambos os modelos, e no Ripple no modelo com mudança de regime.

A atenção está voltada para a possibilidade e quantidade de ocorrências de perdas financeiras superiores às estimativas geradas através dos modelos. Através da inspeção visual pode-se notar que o MS-EGARCH superou os modelos do tipo EGARCH em 1\%, indicando que este modelo é o mais adequado para estimação de VaR no quantil extremo de $1 \%$, ou seja, o modelo com mudança de regime realizou uma previsão mais próxima da perfeição. Em 5\% a ocorrência de perdas foi similar entre os modelos, independentemente do número de regimes houve uma superestimação de $\mathrm{VaR}$, ou seja, ocorreram violações entre as perdas esperadas e previstas. A variação do percentil e a mudança de regime parecem fundamentais para análise de risco já que as previsões em 1\% apresentaram melhor adaptação. Ardia et al. (2019) já haviam documentado evidências de que os modelos com mudança de regime superam os modelos de regime único na previsão de VaR do Bitcoin, resultado também encontrado por Caporale e Zekokh (2019).

A partir dos resultados obtidos, essa pesquisa contribui ampliando uma literatura restrita ao Bitcoin, agora a uma proporção mais representativa desse mercado. Além disso, inclui evidências capturadas exclusivamente pelo modelo MS-EGARCH. Em termos práticos, a contribuição das previsões está relacionada à habilidade de se prever as possíveis quedas ou altas na volatilidade das criptomoedas, ajudando seus investidores e gestores de carteira a obter maior controle sobre seu capital, bem como na tentativa de evitar perdas bruscas. 
(a) Bitcoin - EGARCH
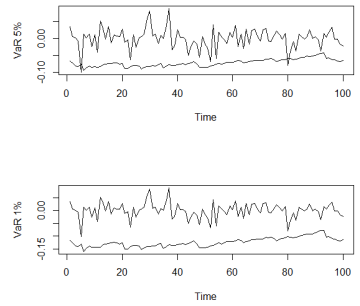

(d) Bitcoin Cash - MS-EGARCH
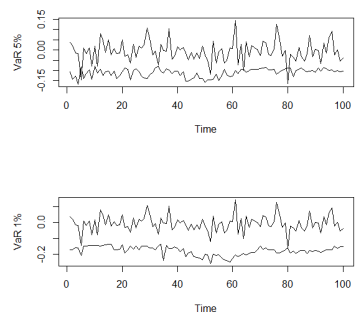

(g) Ethereum - EGARCH
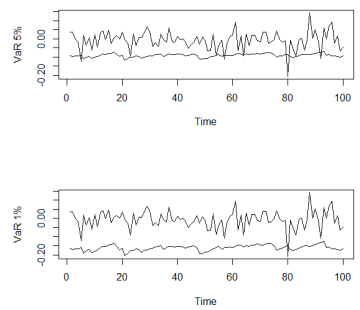

(j) Ripple - MS-EGARCH
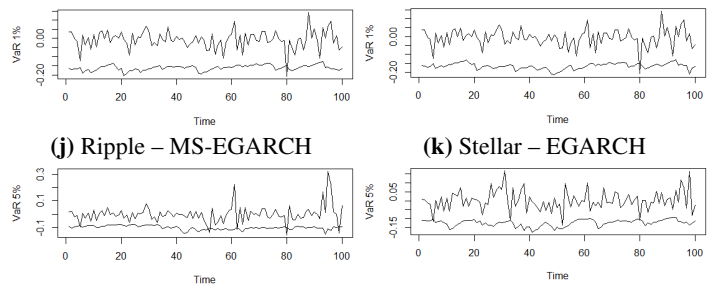

(k) Stellar - EGARCH
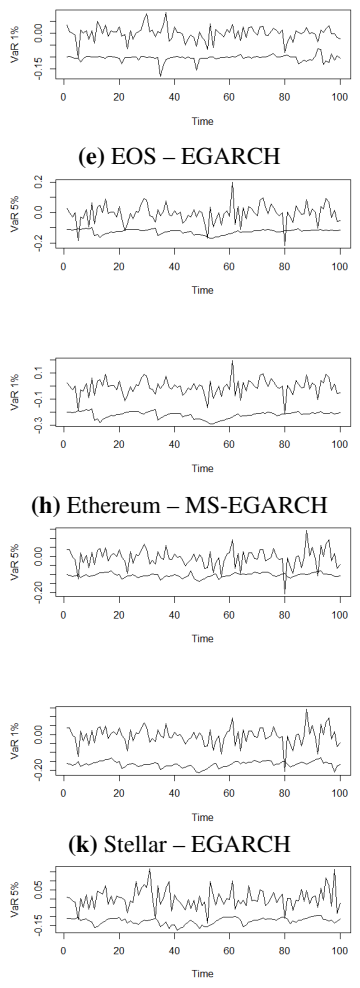

(e) EOS - EGARCH
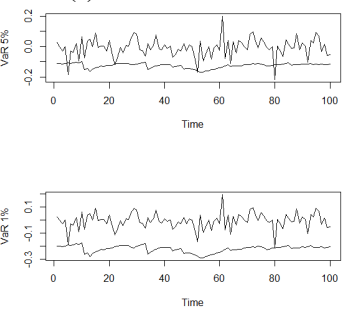

(h) Ethereum - MS-EGARCH

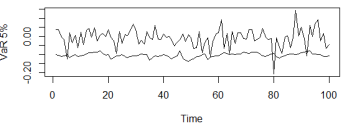

(c) Bitcoin Cash - EGARCH

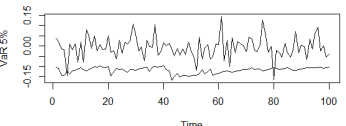

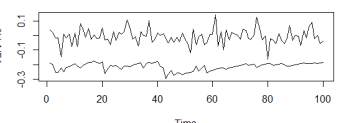

(f) EOS - MS-EGARCH
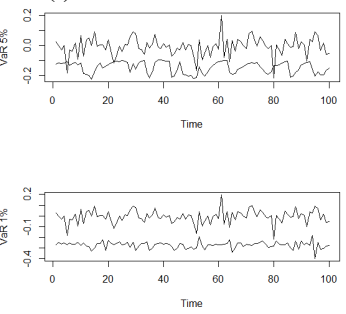

(i) Ripple - EGARCH
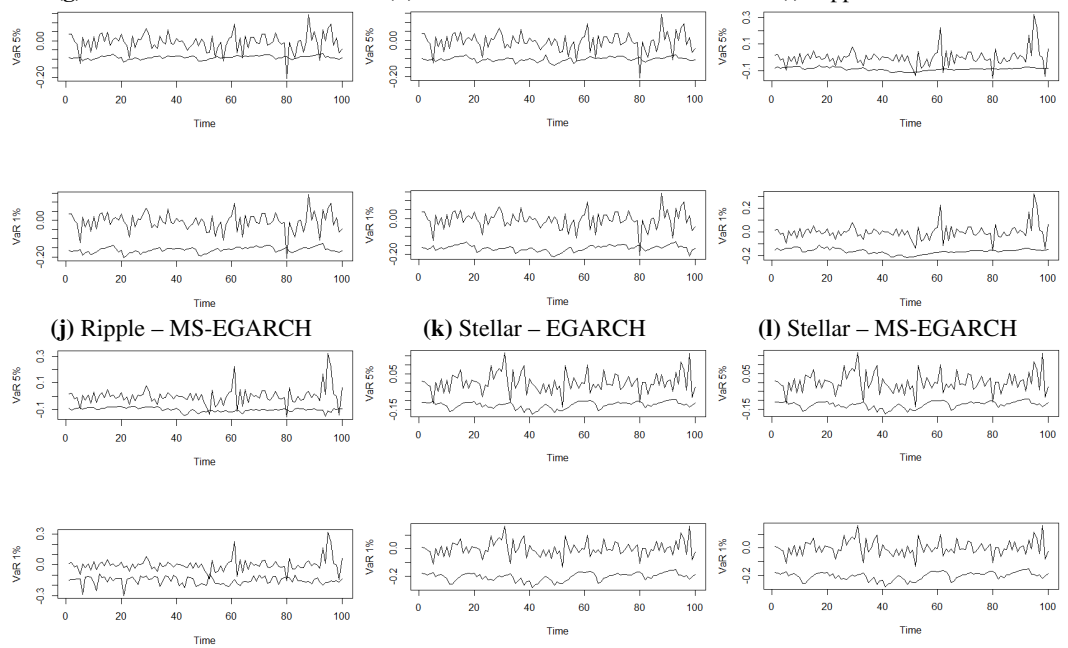

(l) Stellar - MS-EGARCH

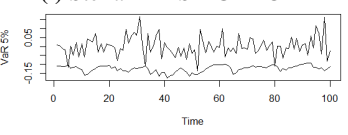

Valores previstos para os 100 passos à frente do VaR considerando $\alpha=5 \%$ e $\alpha=1 \%$. 


\section{Conclusão}

Essa pesquisa teve como objetivo compreender o comportamento volátil em seis criptomoedas de grande representatividade entre 2017 e 2018. Para tal, foram utilizados os modelos EGARCH e MS-EGARCH combinados com diferentes distribuições de probabilidade estatística. Além disso, a capacidade preditiva desses modelos foi avaliada por meio da previsão de VaR. Esse estudo demonstrou que os retornos diários das criptomoedas analisadas apresentam claramente mudanças de regime em sua dinâmica de volatilidade.

$\mathrm{Na}$ análise in-sample, o modelo com mudança de regime confirma a existência de dois estados: o primeiro é caracterizado por um maior efeito $\mathrm{ARCH}$ e menos afetado por assimetrias, enquanto o segundo revela maior efeito da chegada de informações, ou seja, é mais sensível à choque assimétricos. Embora todas as criptomoedas tenham sido influenciadas pela volatilidade passada, essa influência variou dependendo da mudança de 1 para 2 estados, bem como do regime no qual a volatilidade estava concentrada. Na análise outsample, as previsões de $\mathrm{VaR}$ do modelo com mudança de regime superam claramente o modelo de regime único em um alpha de 1\%. A análise também sugere que o uso de modelos EGARCH de regime único podem gerar previsões limitadas de $\mathrm{VaR}$, resultando em um gerenciamento de riscos ineficaz e possíveis falhas na otimização portfólios.

Estes resultados ampliam a compreensão de como diferentes regimes podem influenciar as moedas digitais em sua dinâmica de volatilidade, bem como a influência desses estados na previsão do valor em risco. A persistência e a assimetria sugeridas pelos resultados fornecem subsídios para que investidores tenham cautela em seus investimentos. A volatilidade da maioria das séries de retornos mostrou fortes sinais de assimetria na volatilidade, indicando que choques negativos e positivos têm impacto diferenciado sobre a volatilidade dos retornos, fatos comprovados pelos modelos com mudança de regime. Verificou-se ainda que choques sobre as séries tendem a repercutir por longos períodos. No entanto, ainda existem vários tópicos a serem analisados. Estudos futuros poderiam comparar outras medidas de risco. Também seria muito promissor o uso de dados intraday.

\section{Agradecimentos}

Os autores agradecem a Coordenação de Aperfeiçoamento de Pessoal de Nível Superior pelo suporte financeiro, e também à equipe editorial e aos revisores anônimos pelas excelentes contribuições que aprimoraram a redação e qualidade deste artigo. 


\section{Referências}

Akaike, H. (1973). Maximum likelihood identification of gaussian autoregressive moving average models, Biometrika 60: 255-265.

Ardia, D., Bluteau, K. e Rüede, M. (2019). Regime changes in Bitcoin GARCH volatility dynamics, Finance Research Letters 29: 266-271.

Balcome, K. e Fraser, I. (2017). Do bubbles have an explosive signature in Markov switching models?, Economic Modelling 66: 81-100.

Barivieira, A., Basgall, M., Hasperué, W. e Naiouf, M. (2017). Some stylized facts of the Bitcoin market, Physica A 484: 82-90.

Baur, D. G., Dimpfl, T. e Kuck, K. (2018). Bitcoin, gold and the US dollar A replication and extension, Finance Research Letters 25: 103-110.

Bensaida, A., Boubaker, S., Nguyen, D. e Slim, S. (2018). Value-at-risk under market shifts through highly flexible models, Journal of Forecasting 37: 790-804.

Bollerslev, T. (1986). Generalized autoregressive conditional heteroskedasticity, Journal of Econometrics 31: 307-327.

Bouoiyour, J. e Selmi, R. (2016). Bitcoin: A beginning of a new phase?, Economics Bulletin 36(3): 1430-1440.

Bouoiyour, J., Selmi, R. e Tiwari, A.-K. (2015). Is bitcoin business income or speculative foolery? New ideas through an improved frequency domain analysis, Annals of Financial Economics 10(1): 1-23.

Bouri, E., Azzi, G. e Dyhrberg, A. H. (2016). On the return-volatility relationship in the Bitcoin market around the price crash of 2013, Economics 41: $1-20$.

Bouri, E., Gupta, R., Tiwari, A. K. e Roubaud, D. (2017). Does Bitcoin hedge global uncertainty? Evidence from wavelet-based quantile-in-quantile regressions, Finance Research Letters 23: 87-95.

Cai, J. (1994). A Markov model of unconditional variance in ARCH, Journal of Business \& Economic Statistics 8: 309-316.

Caporale, G. M. e Zekokh, T. (2019). Modelling volatility of cryptocurrencies using Markov-switching GARCH models, Research in International Business and Finance 48: 143-155. 
Catania, L. e Grassi, S. (2017). Modelling crypto-currencies financial timeseries. Working paper.

Chan, S., Chu, J., Nadarajah, S. e Osterrieder, J. (2017). A statistical analysis of cryptocurrencies, Journal of Risk and Financial Managment 10(12): 123.

Christoffersen, P. F. (1998). Evaluating interval forecasts, International Economic Review 4: 841-862.

Chu, J., Chan, S., Nadarajah, S. e Osterrieder, J. (2017). GARCH modelling of cryptocurrencies, Journal of Risk and Financial Management 10(4): 215.

Dendramis, Y., Spungin, G. E. e Tzavalis, E. (2014). Forecasting VaR models under different volatility processes and distributions of return innovations, Journal of Forecasting 33(7): 515-531.

Ding, Z., Granger, C. e Engle, R. (1993). A long memory property of stock market returns and a new model, Journal of Empirical Finance 1: 83-106.

Dyhrberg, A. H. (2016). Bitcoin, gold and the dollar - A GARCH volatility analysis, Finance Research Letters 16: 85-92.

Emiliano, P. C., Vivanco, M. J. F. e Menezes, F. S. (2014). Information criteria: How do they behave in different models?, Computational Statistics \& Data Analysis 69: 141-153.

Engle, R. (1982). Autoregressive conditional heteroscedasticity with estimates of the variance of United Kingdom inflation, Econometrica 50: 9871007.

Engle, R. F. e Ng, V. K. (1993). Measuring and testing the impact of news on volatility, Journal of Finance 48: 1749-1778.

Engle, R. e Manganelli, S. (2004). CAViaR: Conditional Autoregressive Value at Risk by Regression Quantiles, Journal of Business \& Economic Statistics 22(4): 367-381.

Francq, C. e Zokaian, J. (2010). Garch Models: Structure, Statistical Inference and Financial Applications, Wiley Chichester, United Kingdom.

Gençay, R., Selçuk, F. e Ulugülyagci, A. (2003). High volatility, thick tails and extreme value theory in Value-at-Risk estimation, Insurance: Mathematics and Economics 33(2): 337-356. 
Giot, P. e Laurent, S. (2004). Modelling daily Value-at-Risk using realized volatility an ARCH type models, Journal of Empirical Finance 11: 379398.

Glosten, L., Jaganathan, R. e Runkle, D. (1993). On the relation between the expected value and the volatility of the nominal excess returns on stocks, Journal of Finance 48: 1779-1801.

Gray, S. F. (1996). Modeling the conditional distribution of interest rates as a regime-switching process, Journal of Financial Economics 42: 27-62.

Hamilton, J. D. (1989). A new approach to the economic analysis of nonstationary time series and the business cycle, Econometrica 57(2): 357-384.

Hamilton, J. D. e Susmel, R. (1994). Autoregressive conditional heteroscedasticity and changes in regime, Journal of Econometrics 64: 307-333.

Hansen, P. e Lunde, A. (2005). A forecast comparison of volatility models: Does anything beat a GARCH(1,1)?, Journal of Applied Econometrics 20(7): 873-889.

Henry, O. (2009). Regime switching in the relationship between equity returns and short-term interest rates in the UK, Journal of Banking \& Finance 33: $405-414$.

Higgins, M. e Bera, A. (1992). A class of nonlinear ARCH models, International Economic Review 33(1): 137-158.

Jorion, P. (2007). Financial Risk Manager Handbook, John Wiley \& Sons, Hoboken (NJ).

Katsiampa, P. (2017). Volatility estimation for Bitcoin: A comparison of GARCH models, Economics Letters 158: 3-6.

Kupiec, P. (1995). Techniques for verifying the accuracy of risk measurement models, Journal of Derivatives 2: 73-84.

Lamoureaux, C. e Lastrappes, W. (1990). Persistence in variance, structural change and the GARCH model, Journal of Business \& Economic Statistics 8: $225-234$.

Laurent, S., Rombouts, J. e Violante, F. (2012). On the forecasting accuracy of multivariate GARCH models, Journal of Applied Econometrics 27(6): 934-955. 
Liu, M. (2000). Modeling long memory in stock market volatility, Journal of Econometrics 99(1): 139-171.

Mandelbrot, B. (1963). The variation of certain speculative prices, Journal of Business 36(4): 394-419.

McAleer, M. e Da Veiga, B. (2008). Forecasting Value-at-Risk with a Parsimonious Portfolio Spillover GARCH (PS-GARCH) model, Journal of Forecasting 27: 1-19.

Mcneil, A., Frey, R. e Embrechts, P. (2005). Quantitative Risk Management: Concepts, Techniques, and Tools, Princenton University Press, United States.

Nelson, D. (1991). Conditional heteroskedasticity in asset return: A new approach, Econometrica 59(2): 347-370.

Osterrieder, J. e Lorenz, J. (2017). A statistical risk assessment of Bitcoin and its extreme tail behavior, Annals of Financial Economics 12(1): 1-19.

Peng, Y., Albuquerque, P., Sá, J., Pádula, A. e Montenegro, M. (2018). The best of two worlds: Forecasting high frequency volatility for cryptocurrencies and traditional currencies with Support Vector Regression, Expert Systems With Applications 97: 177-192.

Phillip, A., Chan, J. S. e Shelton, P. (2018). A new look at cryptocurrencies, Economics Letters 163: 6-9.

Sajjad, R., Coakley, J. e Nankervis, J. (2008). Markov-switching GARCH modelling of Value-at-Risk, Studies in Nonlinear Dynamics \& Econometrics 12(3): 1-32.

Silva, P., Klotzle, M. C., Pinto, A. e Gomes, L. L. (2019). Volatility estimation for cryptocurrencies using Markov-switching GARCH models, Internation Journal of Financial Markets and Derivatives 7: 1-14.

Su, J. e Hung, J. (2011). Empirical analysis of jump dynamics, heavy tails and skewness on Value-at-Risk estimation, Economic Modeling 28(3): 11171130 .

Sun, P. e Zhou, C. (2014). Diagnosing the distribution of GARCH innovations, Journal of Empirical Finance 29: 287-303.

Trucíos, C. (2019). Forecasting Bitcoin risk measures: A robust approach, International Journal of Forecasting 35: 836-847. 
Trucíos, C., Tiwari, A. e Alqahtani, F. (2019). Value-at-risk and expected shortfall in cryptocurrencies' portfolio: A vine copula-based approach, $A p$ plied Economics pp. 1-15.

Tsay, R. S. (2010). Analysis of Financial Time Series, John Wiley \& Sons.

Tsay, R. S. (2013). An Introduction to Analysis of Financial Data with R, John Wiley \& Sons.

Vandezande, N. (2017). Virtual currencies under EU anti-money laundering law, Computer Law \& Security Review 33: 341-353.

Wennström, A. (2014). Volatility Forecasting Performance: Evaluation of GARCH type Volatility Models on Nordic Equity Indices, Master's thesis, Department of Mathematics, Royal Institute of Technology, Stockholm, Sweden.

Yermack, D. (2015). Is Bitcoin a real currency? An economic appraisal, in D. Lee Kuo Chuen (ed.), Handbook of Digital Currency, Academic Press, San Diego, pp. $31-43$.

Zakoian, J. (1994). Threshold heteroskedastic models, Journal of Economic Dynamics and Control 18: 931-955. 